\title{
Age-related differences in auditory processing as assessed by amplitude-modulation following responses in quiet and in noise
}

\author{
Aravindakshan Parthasarathy ${ }^{1}$, Paul A. Cunningham ${ }^{2}$ and Edward L. Bartlett ${ }^{1,2 *}$ \\ 1 Department of Biological Sciences, Purdue University, West Lafayette, IN, USA \\ 2 Weldon School of Biomedical Engineering, Purdue University, West Lafayette, IN, USA
}

\section{Edited by:}

Antonio Camins, University of

Barcelona, Spain

Reviewed by:

Antonio Camins, University of

Barcelona, Spain

Manuel S. Malmierca, University of

Salamanca, Spain

*Correspondence:

Edward L. Bartlett, Weldon School of Biomedical Engineering, Purdue

University, 206 S. Martin Jischke Drive, West Lafayette, IN 47906, USA.

e-mail: ebartle@purdue.edu
Our knowledge of age-related changes in auditory processing in the central auditory system is limited, unlike the changes in the peripheral hearing organs which are more extensively studied. This study aims to further understanding of temporal processing in aging using non-invasive electrophysiological measurements in a rat model system. Amplitude modulation following responses (AMFRs) were assessed using sinusoidally amplitude modulated (SAM) tones presented to aged (92- to 95-weeks old) and young (9- to 12-weeks old) Fischer-344 rats. The modulation frequency and sound level were systematically varied, and the SAM stimuli were also presented simultaneously with wideband background noise at various levels. The overall shapes and cutoff frequencies of the AMFR temporal modulation transfer functions (tMTFs) were similar between young and aged animals. The fast Fourier transform (FFT) amplitudes of the aged animals were similar to the young in the $181-512 \mathrm{~Hz}$ modulation frequency range, but were significantly lower at most modulation frequencies above and below. There were no significant age-related differences in the nature of growth or FFT amplitudes with change in sound level at 256 and $1024 \mathrm{~Hz}$ modulation frequencies. The AMFR amplitudes were also not correlated with the ABR wave I or wave III amplitudes elicited for broadband click stimuli presented at the same sound level suggesting that sustained AMFR provide complementary information to phasic ABR responses. The FFT amplitudes varied significantly between young and aged animals for SAM stimuli in the presence of background noise, depending on the modulation frequency used and signal to noise ratio. The results show that the representation of temporally modulated stimuli is similar between young and aged animals in quiet listening conditions, but diverges substantially with the addition of background noise. This is consistent with a decrease in inhibition causing altered temporal processing with age.

Keywords: aging, auditory, amplitude modulation, following response, colliculus, evoked potential, Fischer-344

\section{INTRODUCTION}

Age-related auditory decline manifests as pathologies of the peripheral hearing organs, as well as central auditory pathway processing deficits (CHABA, 1988). Studies have shown that perception of isolated speech sounds is not substantially impaired in elderly with moderate presbycusis (Schneider et al., 2005), so simple assessments of hearing thresholds or speech perception in quiet may not detect auditory deficits that would affect quality of life. Listening difficulties arise because people are not just trying to identify isolated speech sounds but rather a stream of speech or other relevant sound, often superimposed upon a background of other talkers or background noise. It is in these more realistic listening conditions that elderly listeners have the most difficulties (Frisina and Frisina, 1997). Loss in temporal processing has been reported from psychophysical studies, even when the elderly listeners have normal audiograms and thresholds comparable to younger listeners (Schneider et al., 1994; Snell et al., 1994; Frisina and Frisina, 1997; Strouse et al., 1998; Mazelova et al., 2002). These studies point to the role the central auditory pathway plays in temporal processing and suggests that this processing degrades with age.
In the auditory pathway, neurons in the auditory brainstem and midbrain nuclei respond to temporal stimuli in a stimulus-synchronized manner (Frisina et al., 1990a,b). These phase locked responses in the brainstem and the midbrain recorded as auditory evoked potentials have developed into indispensable diagnostic tools for assessing hearing loss and auditory neural processing changes (Starr et al., 1996; Hood, 1998; King et al., 2002; Russo et al., 2004). Auditory brainstem responses (ABRs) are the predominant physiologic measure of hearing sensitivity and are typically evoked by brief click or tone stimuli (Hall, 2006). However, many speech sounds and other behaviorally relevant sounds last for tens to hundreds of milliseconds and contain periodic components (Rosen, 1992). Such stimuli can be tracked using either the frequency following response (FFR) to track low carrier frequencies or the amplitude modulation following response (AMFR) to track changes in the modulation envelope (Picton et al., 2003). The FFR and the AMFR are the summed synchronized population responses of spiking activity and synaptic potentials of neurons in the auditory brainstem and midbrain (Herdman et al., 2002). 
Recent studies have used the FFR and AMFR in order to analyze central auditory processing non-invasively in humans with auditory processing deficits. Studies on elderly humans found that there were no significant differences in AMFR for slow AM frequencies $(<50 \mathrm{~Hz})$ even when modulation depth was changed or a high frequency background noise was added (Boettcher et al., 2001). Modulation frequencies of $90 \mathrm{~Hz}$ were shown to elicit fewer phase locked responses in the aged as compared to young (Leigh-Paffenroth and Fowler, 2006). Other recent studies have examined the use of AMFR in assessing neural population activity in response to speech sounds in adults, children, and children with specific language impairment (SLI) (Cunningham et al., 2001; King et al., 2002; Krishnan, 2002; Russo et al., 2005). It was found that children with SLI exhibited degraded FFR responses to the vowel-consonant-vowel /ada/ presented with background noise. The largest degradations occurred when FFR responses to stimuli in the $450-750 \mathrm{~Hz}$ range were analyzed.

Though AMFRs have been used extensively to characterize the auditory system in many animal species (Dolphin and Mountain, 1992; Finneran et al., 2007; Henry and Lucas, 2008; Mooney et al., 2009), very few studies, if any, have looked at the change in AMFRs with age in an animal model. Most aging studies in animals examine the differences in ABR properties, in response to clicks and pure tones, or gap detection paradigms (Backoff and Caspary, 1994; Boettcher et al., 1995, 1996). The present study will investigate changes in AMFR in the Fischer-344 rat, where there is information about age-related hearing deficits in single neuron response properties (Palombi and Caspary, 1996; Caspary et al., 2005; Schatteman et al., 2008), cell function and morphology (Helfert et al., 1999, 2003; Meng et al., 2007) as well as peripheral changes in the cochlea and hair cells (Popelar et al., 2003; Buckiova et al., 2007; Chen et al., 2009). This will enable a more complete understanding of the changes in auditory processing with age.

At the anatomical level, one consistent finding across central auditory regions is that the number of inhibitory glycine and $\mathrm{GABA}_{\mathrm{A}}$ receptors decline with age (Caspary et al., 1990a,b; Helfert et al., 1999; Ling et al., 2005; Frisina and Walton, 2006). GABA and glycine have been specifically shown to shape response curves of neurons in the central auditory system to complex temporally modulated stimuli (reviewed in Caspary et al., 2008), in the cortex (Wang et al., 2000, 2002; Foeller et al., 2001), the inferior colliculus (Caspary et al., 2002), and the dorsal cochlear nucleus (Backoff et al., 1999; Schatteman et al., 2008). Given this decrease in inhibition, there are at least two competing hypotheses to test with regards to interpreting population AMFR to temporal modulation in aged subjects. One hypothesis is that decreased inhibition will lead to no change or increased AMFR amplitudes at the tested AM modulation frequency in aged subjects. This lack of effect of age has been observed in human AMFR (Boettcher et al., 2001), but the modulation frequency used was relatively low, so inhibition may not be necessary to maintain temporal precision. A competing hypothesis is that the response amplitudes will be similar, but the decreased inhibition will allow less precision in the responses and lead to a smaller response peak at the modulation frequency. An extension of this hypothesis is that the reduced precision will only be evident under difficult listening conditions, such as in the presence of background noise, assuming the excitatory inputs are well synchronized in quiet conditions when stimulus envelopes are fully intact. This has been observed in human psychophysical studies, where older adults with normal hearing exhibit reduced speech recognition in noise, even when their speech recognition in quiet is comparable to younger adults with normal hearing (Dubno et al., 1984).

In this study, ABR and AMFRs were compared between young and aged Fischer-344 rats. AMFRs were obtained for sinusoidally amplitude modulated (SAM) tones to obtain a temporal modulation transfer function (tMTF) and determine whether there are changes in frequency cutoffs with age. Based on the AMFR amplitudes from the tMTF, the sound levels of two modulation frequencies were varied to determine an AMFR amplitude-level function. SAM stimuli were then presented simultaneously with wide band noise (WBN) to determine whether temporal processing of these stimuli change in degraded listening conditions.

\section{MATERIALS AND METHODS SUBJECTS}

Ten young adult (9- to 12-weeks old, weighing $\sim 275 \mathrm{~g}$ ) and ten aged (92- to 95-weeks old, weighing $\sim 450 \mathrm{~g}$ ) Fischer-344 rats obtained from Taconic, were used in this study. The animals were housed in the animal care facility for the period of the study in quiet conditions. All protocols are approved by the Purdue animal care and use committee (PACUC).

\section{EXPERIMENTAL SETUP}

Experiments were performed in a $9^{\prime} \times 9^{\prime}$ double walled acoustic chamber (Industrial Acoustics Corporation). The animals were anesthetized using isoflurane gas anesthesia. The induction took place in a separate induction chamber at $4 \%$ concentration of isoflurane. The animals were then transferred to the manifold, and the experiment run at $1.25-1.5 \%$ concentration of isoflurane anesthesia for young animals and $1-1.25 \%$ for the aged. The animals were placed on a water circulated warming blanket (Gaymar) set to $37^{\circ} \mathrm{C}$ with the pump placed outside the recording chamber to eliminate audio and electrical interferences. The stimulus was presented free field using the speaker to the right ear of the animal, at a distance of $115 \mathrm{~cm}$ from speaker to ear, with the sound level calibrated to this distance using a Bruel Kjaer microphone. The positive electrode was placed on the forehead of the animal along the midline from the $\mathrm{Cz}$ to the $\mathrm{Fz}$ position, the negative electrode was placed under the right ear, along the mastoid, and the ground electrode was placed in the nape of the neck. Impedances were always less than $1 \mathrm{~K}$ as tested using the head-stage (RA4LI, Tucker Davis technologies, or TDT). Control recording setups were recreated with a recently deceased animal using the maximum sound levels to ensure that there was no stimulus bleed-through or other electrical or mechanical interferences, so all reported data obtained were physiological in nature.

Sounds were generated by SigGenRP (TDT). Signal presentation and acquisition was done by BioSig software (TDT). Waveforms were converted to sounds and delivered via a multichannel processor (RX6, TDT) through a Bowers and Wilkins DM601 speaker. The output from the speakers was calibrated using a Bruel Kjaer microphone and SigCal (TDT), and was found to be within $\pm 6 \mathrm{~dB}$ for the frequency range tested. ABRs and AMFRs were recorded using 
sub-dermal needle electrodes (Ambu) connected to a low-impedance head-stage (RA4LI, TDT) and amplified (RA4PA preamplifier, TDT). Digitized waveforms and spike times are recorded with a multichannel recording and stimulation system (RX7-2, TDT, and RZ-5, TDT) and analyzed with BioSig or MATLAB.

\section{STIMULUS DESCRIPTION AND RECORDING PROCEDURES}

Auditory brainstem responses were recorded using broadband rectangular click stimuli of alternating polarity, 0.1-ms long, presented at 26.6 clicks/s. The acquisition window was $20 \mathrm{~ms}$ and each ABR was an average of 1500 repetitions. The intensity of the clicks was reduced in 10-dB steps starting from $95 \mathrm{~dB}$ down to $5 \mathrm{~dB}$ to obtain the click threshold.

Sinusoidally amplitude modulated tones were 200-ms long with a 5 -ms cosine squared ramp at onset and offset and played at a rate of 3.1/s. All stimuli were played at $30 \mathrm{~dB}$ above click ABR threshold. The acquisition window was 300-ms long and each AMFR was an average of 200 repetitions which was determined empirically to produce a reliable response. The carrier frequency of the SAM stimuli was $8 \mathrm{kHz}$. This frequency is in the most sensitive region of the rat IC (Hernandez et al., 2005), and has been shown to be minimally affected by hair cell loss with age (Syka, 2010). For testing the increase in AMFR amplitude with sound level, the intensity of the SAM stimuli was varied from 95 to $5 \mathrm{~dB}$ in $10 \mathrm{~dB}$ steps for 256 and $1024 \mathrm{~Hz}$ modulation frequencies. These were chosen to be one octave above and below the peak modulation frequency $(512 \mathrm{~Hz})$ for which the most robust responses were observed. For calculating the tMTF, the modulation frequency was increased from 32 to $4096 \mathrm{~Hz}$ in half octave steps, at a sound level $30 \mathrm{~dB}$ above click threshold. To test AMFR to SAM stimuli in WBN, the SAM stimuli of the three modulation frequencies, 256, 512, and $1024 \mathrm{~Hz}$ were played at $30 \mathrm{~dB}$ above threshold, and the WBN was varied from $+30 \mathrm{~dB}$ signals to noise ratio (SNR) to $-10 \mathrm{~dB}$ SNR.

\section{DATA ANALYSIS}

The data collected were filtered from 30 to $3000 \mathrm{~Hz}$ or 30 to $4500 \mathrm{~Hz}$ (when using modulation frequencies higher than $2048 \mathrm{~Hz}$ ) using BioSig software (TDT). Filtered data were exported to MATLAB. For ABRs, first and third wave amplitudes were computed using BioSig software and custom written programs in MATLAB. For AMFRs, fast Fourier transforms (FFT) were performed on timedomain waveforms obtained from BioSig, 10-190 ms after stimulus onset to exclude the ABR transients, using custom programs written in MATLAB. An example of the time-domain waveform used to calculate the FFTs are shown in Figure 1 for young and aged animals. This particular waveform was evoked by $8-\mathrm{kHz}$ tones modulated at $256 \mathrm{~Hz}$ SAM at $75 \mathrm{~dB}$. The response waveforms show clear periodic amplitude modulations that are at the SAM frequency. The maximum energy at modulation frequency or one frequency bin ( $1 \mathrm{~Hz}$ ) above and below it gave the peak FFT amplitude. The noise floor was calculated as the average of five frequency bins ( $1 \mathrm{~Hz}$ each) above and five bins below the central three bins.

\section{STATISTICAL ANALYSIS}

Repeated measures ANOVAs (MANOVA) were performed to analyze the FFT amplitudes using custom scripts in SAS (Proc MIXED; SAS Institute Inc., v. 9.1, Cary, NC, USA). A log transformation was applied to the FFT amplitudes to produce a normally distributed

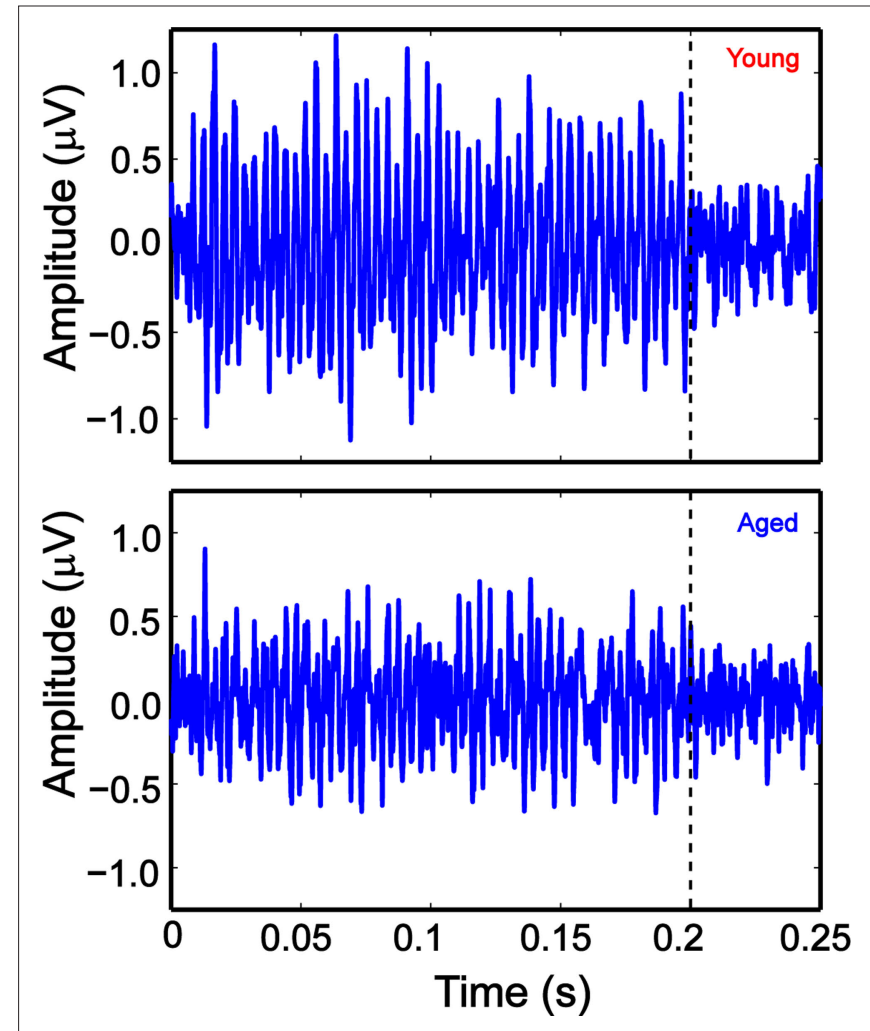

FIGURE 1 | Example AMFR traces of young (top panel) and aged (bottom panel) animals, at $256 \mathrm{~Hz} \mathrm{AM}, 100 \%$ modulation depth and $75 \mathrm{~dB}$ SPL, showing clear phase locking to the AM envelope of the stimulus. The stimulus was 200-ms long, and the recording window was $300 \mathrm{~ms}$. The dotted lines indicate stimulus offset. The $x$ axis shows the time of acquisition and the $y$ axis shows the amplitude of the AMFR trace.

data set (Proc UNIVARIATE; SAS). The ANOVA for the tMTF consisted of the main effects of age and modulation frequency, as well as their interaction. The ANOVAs for experiments using varying levels of SAM stimuli, as well as WBN consisted of three main effects each - age, modulation frequency, and level or age, modulation frequency, and SNR respectively, as well as all their two-way and three-way interactions. Sound levels from 44 to $94 \mathrm{~dB}$ were considered for the SAM level experiment in the statistical analysis. This excluded a significant proportion of sub-threshold data, especially for older animals, and resolved the differences at the higher sound levels more clearly. The factors were analyzed for simple effects (for two-way and three-way interactions) as well as comparison of least squares (LS) mean. Normal probability plots of the residuals indicated that they were normally distributed for each ANOVA model (proc UNIVARIATE). Significant effects were reported using the LS means and their differences, with a 95\% confidence interval. LS mean \pm SE are reported throughout unless otherwise specified.

\section{RESULTS}

SAM TONES WITH VARYING MODULATION FREQUENCIES: OBTAINING THE TEMPORAL MODULATION TRANSFER FUNCTION

Sinusoidally amplitude modulated $8 \mathrm{kHz}$ carriers presented at $30 \mathrm{~dB}$ above click threshold and 100\% modulation depth were used to generate a modulation transfer function for the AMFR. 
The modulation frequency range of $32-4096 \mathrm{~Hz}$ was tested in half octave steps. AMFR amplitudes were largest from 181 to $512 \mathrm{~Hz}$. Above this frequency range, AMFR amplitudes decreased rapidly, but clear FFT peaks were observed until $2048 \mathrm{~Hz}$ in young animals and $1448 \mathrm{~Hz}$ in aged animals (Figure 2). For modulation frequencies in the 32-64 Hz range, FFT peaks were clearly observed in some but not all animals, as surrounding frequencies had similar FFT amplitudes resulting from a general high energy region. Overall, the shape and nature of the tMTF was similar between young and aged animals, for SAM stimuli presented in quiet, at 100\% modulation depth.

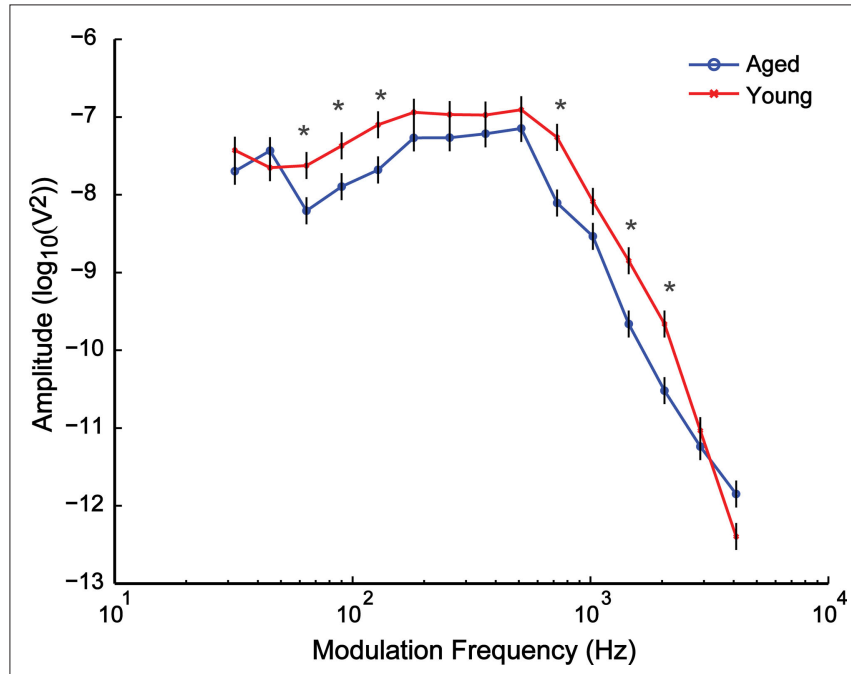

FIGURE 2 | Similarity in overall shape and nature of the curves was observed when comparing the temporal modulation-transfer function (tMTF) of young and aged animals. The $x$ axis shows the modulation frequencies in a logarithmic scale, and the $y$ axis shows the least squares means (LSM) \pm SE of the FFT amplitudes. Asterisks indicate statistically significant difference in LSMs $(P<0.05$, MANOVA).
Statistical analysis showed significant main effects of age $\left(F_{1,18}=12.33, P=0.0025\right)$ and modulation frequency $\left(F_{14,252}=192.18\right.$, $P<0.0001)$ as well as a significant interaction effect of age $\times$ modulation frequency $\left(F_{14,252}=2.76, P=0.0008\right)$. The AMFR amplitudes between the young and the aged animals were significantly different $(P<0.05)$ for most modulation frequencies below $181 \mathrm{~Hz}$ and above $1024 \mathrm{~Hz}$, but there was no significant difference in the amplitudes in the 181-512 Hz range and at $1024 \mathrm{~Hz}$ (Figure 2).

\section{SAM STIMULI WITH VARYING SOUND LEVEL}

After determining the tMTF, the relationship of the AMFR amplitude with respect to level was tested by varying the sound level of the SAM tone from 14 to $94 \mathrm{~dB}$ SPL in $10-\mathrm{dB}$ steps. AMFRs to SAM stimuli with two modulation frequencies, 256 (Figure 3A) and $1024 \mathrm{~Hz}$ (Figure 3B), were obtained from young and aged animals at different sound levels. When comparing the absolute FFT amplitudes, both young and aged animals show a monotonic increase in FFT amplitudes up until $74 \mathrm{~dB}$ in the young and $84 \mathrm{~dB}$ in the aged. Responses decreased for stimuli presented at 84 and $94 \mathrm{~dB}$ in the young and $94 \mathrm{~dB}$ in the aged. The overall trend for changes in FFT amplitudes with increasing sound levels was similar between young and aged animals, as witnessed by the similar shape of the response curves.

Analysis of the absolute FFT amplitudes showed significant main effects of age, modulation frequency and intensity as well as significant effects in all two-way and three-way interactions (Table 1). The amplitudes of the AMFRs were significantly higher in younger animals at $256 \mathrm{~Hz}$ modulation frequency for all sound levels below $84 \mathrm{~dB}(P<0.05)$, but were not significantly different at 84 and $94 \mathrm{~dB}$. At $1024 \mathrm{~Hz}$, there were no significant differences in AMFR amplitudes at sound levels below $74 \mathrm{~dB}$ or at the highest sound level $(94 \mathrm{~dB})$ but showed a significant difference at 74 and $84 \mathrm{~dB}$ where AMFR amplitudes were the largest.

When the same responses were analyzed relative to the ABR threshold, a different picture emerged. Figures 4 A,B shows a comparison between the FFT amplitudes relative to threshold between

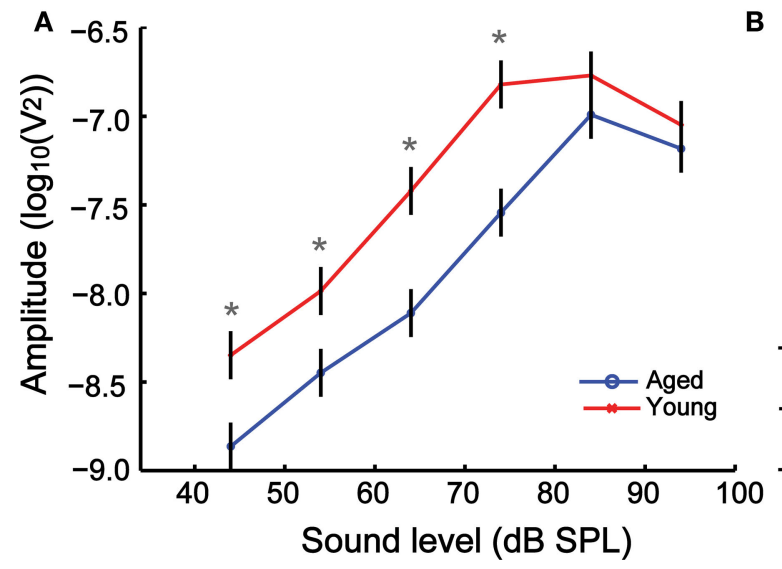

FIGURE 3 |The growth of AMFR amplitudes with sound level is similar between young and aged animals at 256 (A) and $1024 \mathrm{~Hz}(\mathbf{B}) \mathrm{AM}$, though the amplitudes are greater for the young animals at most sound levels. The sound

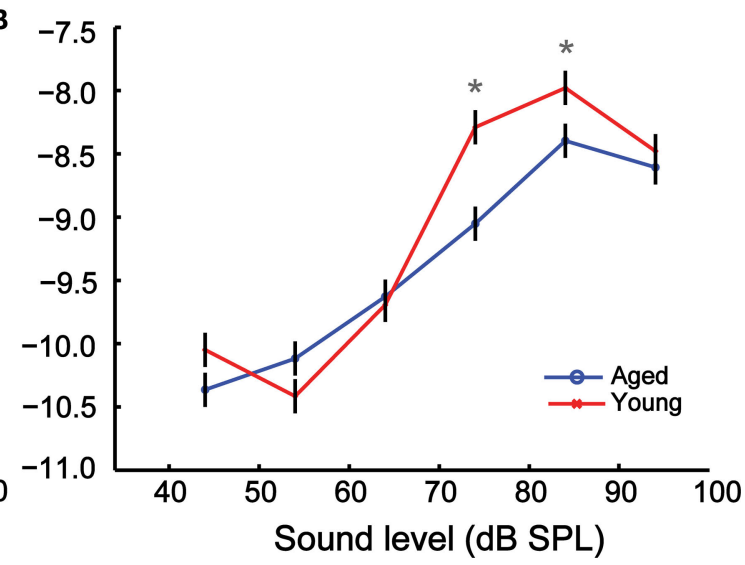

level of the AM stimuli is represented along the $x$ axis, and the $\mathrm{LSM} \pm \mathrm{SE}$ of the FFT amplitudes along the $y$ axis. Asterisks indicate statistically significant difference in LSMs $(P<0.05$, MANOVA). 
young and aged animals for 256 and $1024 \mathrm{~Hz}$ modulation frequencies respectively. There were no significant differences between the FFT amplitudes of the young and aged at most sound levels and either modulation frequency. The relative FFT amplitudes increased with sound level similarly between young and aged animals. Analysis of the relative FFT amplitudes showed significant main effects of sound level and modulation frequency but no significant effects of age. The two-way interaction between sound level and modulation frequency was significant, but all other two-way and three-way interactions involving age were not significant (Table 2).

\section{AUDITORY BRAINSTEM RESPONSES AND THEIR CORRELATION WITH AMFRs}

In order to relate AMFR to more conventional ABRs and to establish appropriate levels for AMFR stimuli, ABRs were measured for young and aged animals using 0.1-ms rectangular click stimuli. Figure 5 shows ABRs from a young (Figure 5A) and aged (Figure 5B) animal at an example sound level. The first positive peak is

Table 1 |Tests of main effects of sound level, age and modulation frequency, as well as all their interaction factors on AMFR amplitudes using repeated measures ANOVA.

\begin{tabular}{llrr}
\hline Effect & DF Num, Den & \multicolumn{1}{l}{$\boldsymbol{F}$} & \multicolumn{1}{c}{$\boldsymbol{P}$} \\
\hline Sound level & 5,90 & 194.43 & $<0.0001$ \\
Age & 1,18 & 8.50 & 0.0092 \\
Modulation frequency (MF) & 1,18 & 1243.07 & $<0.0001$ \\
Sound level $\times$ Age & 5,90 & 4.37 & 0.0013 \\
Sound level $\times$ MF & 5,90 & 6.44 & $<0.0001$ \\
Age $\times$ MF & 1,18 & 7.19 & 0.0152 \\
Sound level $\times$ Age $\times$ MF & 5,90 & 3.35 & 0.0080
\end{tabular}

DF, degrees of freedom; Num, numerator; Den, denominator. usually designated as wave I and the third and largest positive peak as wave III (Backoff and Caspary, 1994). The mean ABR threshold of the young was $47 \pm 4.22 \mathrm{~dB}$ and that of the aged was $55 \pm 4.71 \mathrm{~dB}$. The thresholds of the aged were significantly higher than the young $(P<0.01$, Mann-Whitney $U$ test $)$. Both the young and the aged showed similar ABR waveform morphologies (Figure 5).

Wave I amplitudes from ABRs presented $30 \mathrm{~dB}$ above threshold were compared with AMFR amplitudes from the same sound level at 256- and $1024-\mathrm{Hz}$ modulation frequencies, to determine whether there was a correlation between the two. As seen in Figure 6, there were no significant correlations between the ABR wave I amplitudes and AMFR amplitudes from SAM stimuli at either 256- (Figure 6A) or 1024- $\mathrm{Hz}$ (Figure 6B) modulation frequency, presented at $30 \mathrm{~dB}$ above ABR threshold. There were also no significant correlations when wave III amplitudes were compared with AMFR amplitudes from the same sound level at 256- or 1024-Hz modulation frequencies, or when the young and

Table 2 |Tests of main effects of sound level (relative to click ABR threshold), age and modulation frequency, as well as all their interaction factors on AMFR amplitudes using repeated measures ANOVA.

\begin{tabular}{llrr}
\hline Effect & DF Num, Den & \multicolumn{1}{l}{$\boldsymbol{F}$} & \multicolumn{1}{c}{$\boldsymbol{P}$} \\
\hline Sound level & 5,78 & 98.18 & $<0.0001$ \\
Age & 1,18 & 0.03 & 0.8686 \\
Modulation frequency (MF) & 1,18 & 516.43 & $<0.0001$ \\
Sound level $\times$ Age & 5,78 & 2.18 & 0.0643 \\
Sound level $\times$ MF & 5,78 & 2.88 & 0.0195 \\
Age $\times$ MF & 1,18 & 1.56 & 0.2278 \\
Sound level $\times$ Age $\times$ MF & 5,78 & 2.08 & 0.0762
\end{tabular}

DF, degrees of freedom; Num, numerator; Den, denominator.
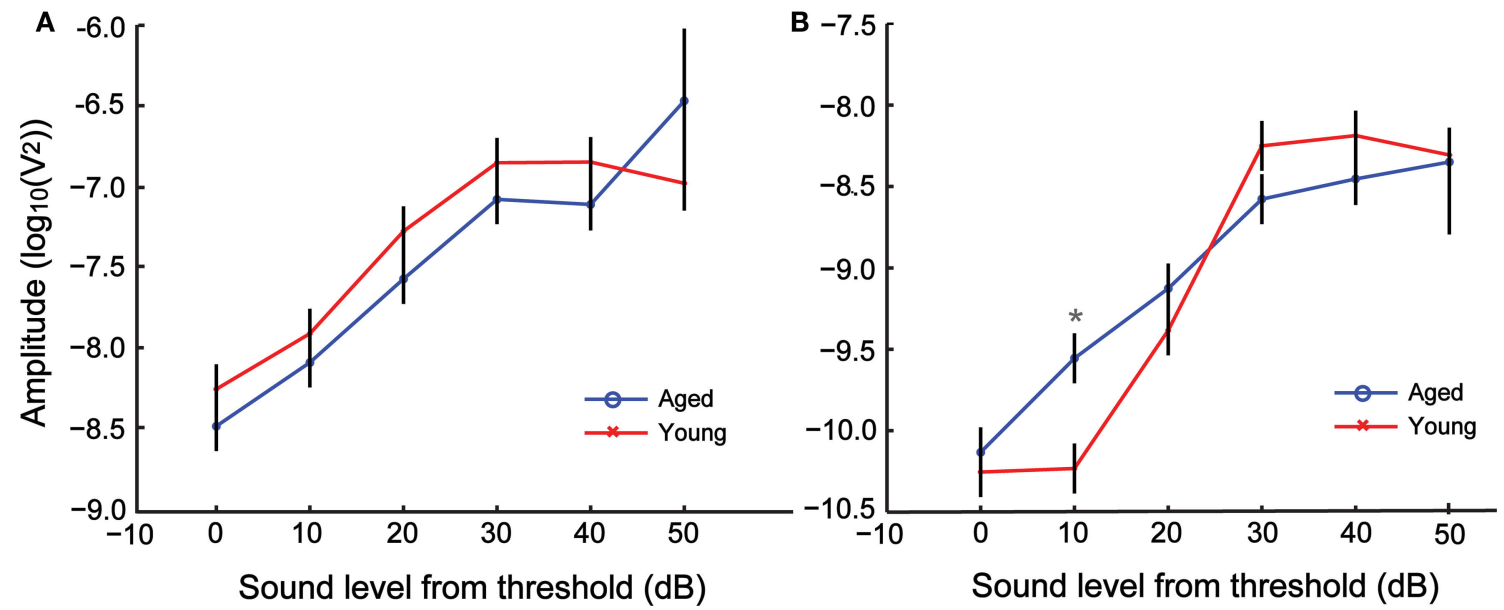

FIGURE 4 |AMFR amplitudes with increase in sound level for 256 (A) and $1024 \mathrm{~Hz}$ (B) AM shows no difference in amplitude between young and aged animals for nearly all sound levels when plotted relative to individual click ABR threshold. The $x$ axis indicates sound level relative to threshold, with 0 representing click $A B R$ threshold. The $y$ axis indicates the LSM \pm SE of the FFT amplitudes. Asterisks indicate statistically significant difference in LSMs $(P<0.05$, MANOVA) 


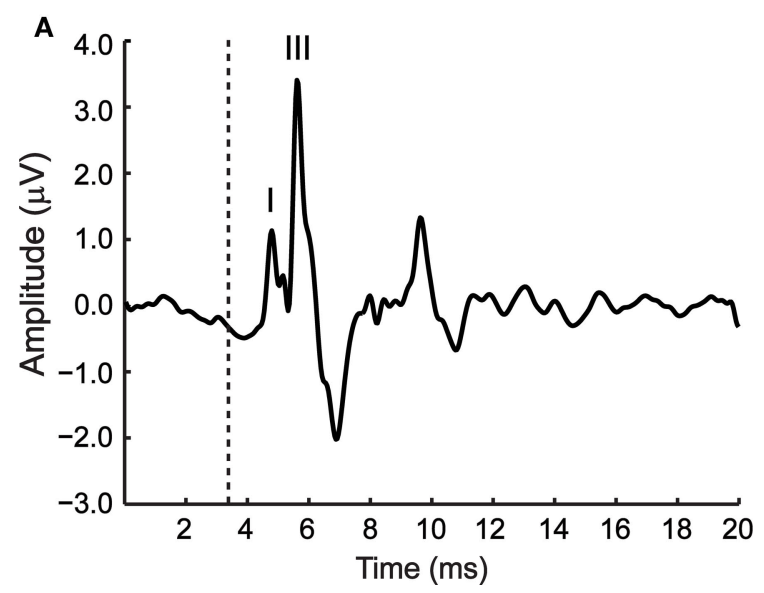

FIGURE 5 | Representative ABR waveforms from a young (A) and aged animal (B), in response to a 0.1-ms rectangular click stimulus presented at $85 \mathrm{~dB}$. The first and third positive peaks are labeled wave I and wave III

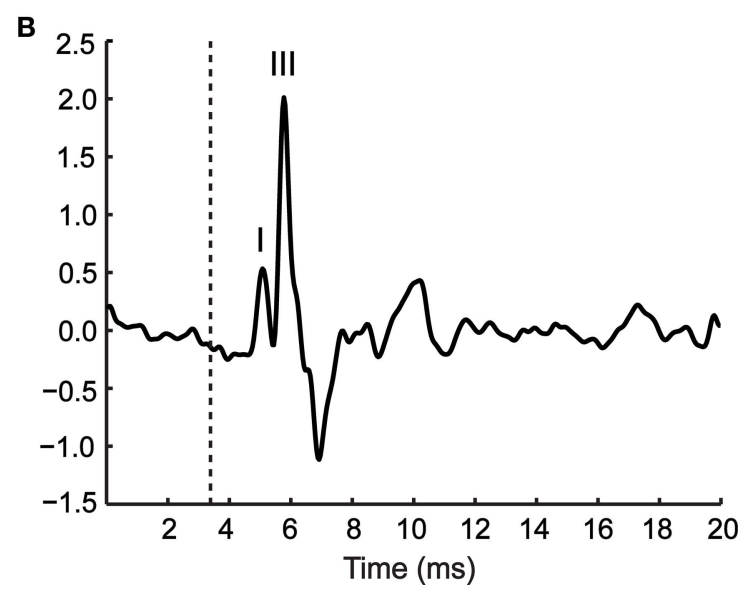

respectively. The dotted line indicates stimulus onset at the ear. The time of the recording window $(20 \mathrm{~ms}$ ) is indicated along the $x$ axis and the amplitude of the ABR along the $y$ axis.
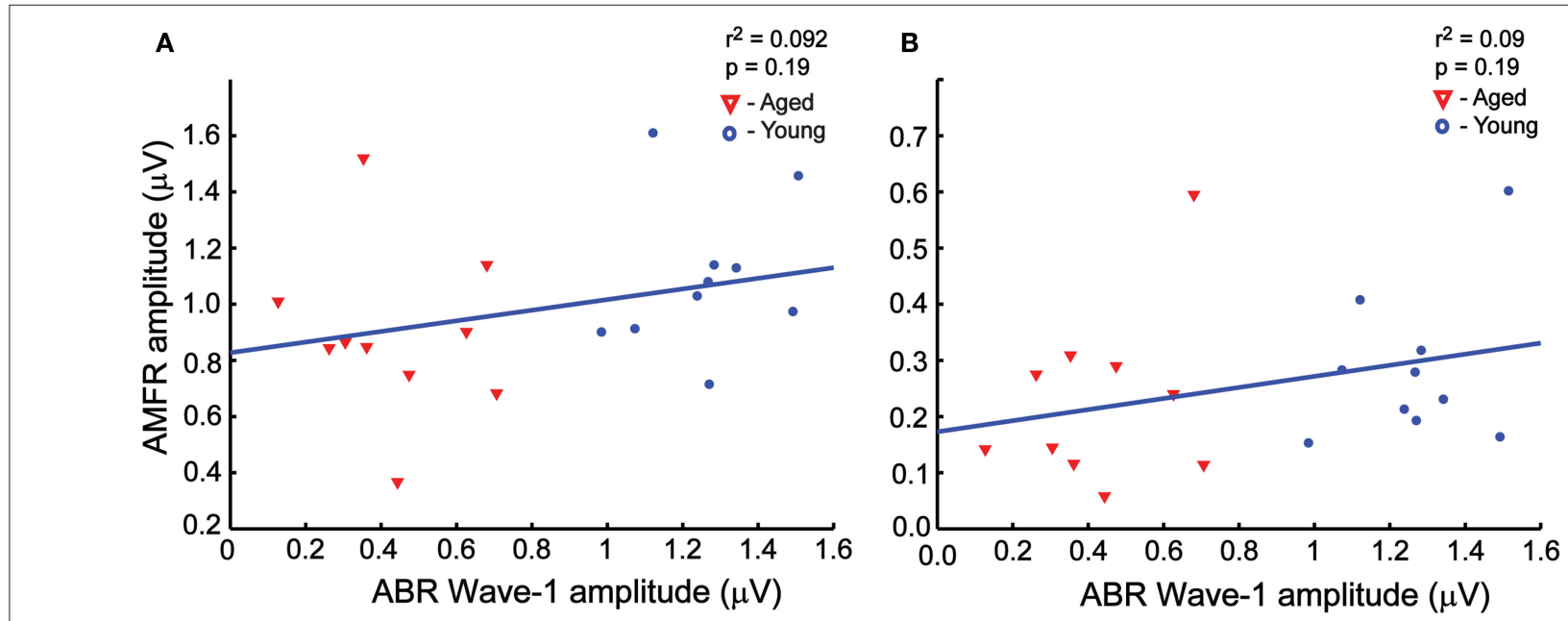

FIGURE 6 |There was no significant correlation between wave I amplitudes and the AMFR amplitudes at $30 \mathrm{~dB}$ above click threshold at both $256 \mathrm{~Hz}\left(\mathrm{~A}, r^{2}=0.092, P=0.19\right)$ and $1024 \mathrm{~Hz}\left(\mathrm{~B}, r^{2}=0.092, P=0.19\right)$ modulation frequencies. The ABR amplitudes for rectangular click stimuli at

$30 \mathrm{~dB}$ relative to threshold is plotted along the $x$ axis, and the AMFR amplitudes for the SAM stimuli at $30 \mathrm{~dB}$ relative to threshold is plotted along the $y$ axis. Similar results were observed for wave III ABR amplitudes vs. AMFR amplitudes (not shown).

the aged populations were compared separately (data not shown). Thus, the AMFR and ABR measurements here provide independent, complementary information about tonic and phasic auditory responses respectively.

\section{SAM IN WIDE BAND NOISE}

In order to investigate temporal coding when the AM envelope is degraded, responses to three modulation frequencies, 256, 512, and $1024 \mathrm{~Hz}$ were recorded in the presence of simultaneously presented WBN. These modulation frequencies were chosen to include one octave above and below $512 \mathrm{~Hz}$, which showed the most robust AMFR in the tMTF. The SAM tones were presented at $30 \mathrm{~dB}$ above click threshold and the WBN level was varied from $+30 \mathrm{~dB}$ SNR to $-10 \mathrm{~dB}$ SNR.
Analysis of the FFT amplitudes showed significant main effects of SNR and modulation frequency, as well as significant effects for all two-way and three-way interactions between SNR, age and modulation frequency (Table 3). There were no significant differences in AMFR amplitudes between the young and the aged at $+30 \mathrm{~dB}$ SNR for any of the modulation frequencies used. For $256 \mathrm{~Hz} \mathrm{AM}$, an increase in noise by $10 \mathrm{~dB}(+20 \mathrm{~dB}$ SNR) produced a substantial decrease in the AMFR amplitudes in the younger animals but little or no decrease in the aged animals. The amplitudes of the young were significantly less than the aged $(P<0.05)$ at $256 \mathrm{~Hz},+20 \mathrm{~dB}$ SNR. This trend further continued at SNR +10 , with the responses in young animals being diminished with noise, and significantly lower than those of the aged animals $(P<0.05)$. At 0 and $-10 \mathrm{~dB}$ SNR, for $256 \mathrm{~Hz}$, the amplitudes of the aged 
decreased sharply and there was no significant difference in the responses between young and aged animals (Figure 7A). Similar results were obtained when using a modulation frequency of $512 \mathrm{~Hz}$, but in this case, the aged and young animals were significantly different only at an SNR of $+10(P<0.05)$, with the aged animals having higher amplitude when compared to the young (Figure 7B). At a modulation frequency of $1024 \mathrm{~Hz}$, the young

Table 3 | Tests of main effects of signal-to-noise ratio (SNR), age and modulation frequency, as well as all their interaction factors on AMFR amplitudes using repeated measures ANOVA.

\begin{tabular}{llrr}
\hline Effect & DF Num, Den & \multicolumn{1}{l}{$\boldsymbol{F}$} & \multicolumn{1}{l}{$\boldsymbol{P}$} \\
\hline Signal to noise ratio (SNR) & 4,72 & 17.53 & $<0.0001$ \\
Age & 1,18 & 0.46 & 0.5070 \\
Modulation frequency (MF) & 2,30 & 77.24 & $<0.0001$ \\
SNR $\times$ Age & 4,72 & 7.52 & $<0.0001$ \\
SNR $\times$ MF & 8,120 & 6.18 & $<0.0001$ \\
Age $\times$ MF & 2,30 & 35.98 & $<0.0001$ \\
SNR $\times$ Age $\times$ MF & 8,120 & 4.82 & $<0.0001$ \\
\hline
\end{tabular}

DF, degrees of freedom; Num, numerator; Den, denominator. and aged had no difference in AMFR amplitudes at +30 and +20 $\mathrm{SNR}$. For $\mathrm{SNRs} \leq 10 \mathrm{~dB}$ the young animals had significantly higher amplitude than the aged $(P<0.05)$, unlike the lower modulation frequencies (Figure 7C).

\section{DISCUSSION}

In this study, the change in AMFR amplitudes with sound intensity is similar for young and aged animals under quiet conditions, especially when the sound levels are considered relative to the threshold of each individual animal. These changes in AMFR amplitudes are also not correlated with the wave I or wave III amplitudes of the ABR, and hence these AMFR amplitudes cannot be predicted simply by measuring the auditory brainstem response. The overall shape of the tMTF is largely comparable between the young and aged animals. The AMFR amplitudes are similar for the young and aged animals in the 181-512 Hz range but are higher for young animals at lower and higher modulation frequencies. This is largely in accordance with human psychophysical studies which have also shown a similarity in the overall shape of the tMTF between aged and young subjects using SAM stimuli presented in quiet, with an age-related decline in envelope detection for modulation frequencies $40-200 \mathrm{~Hz}$ (He et al., 2008). Older listeners also have more

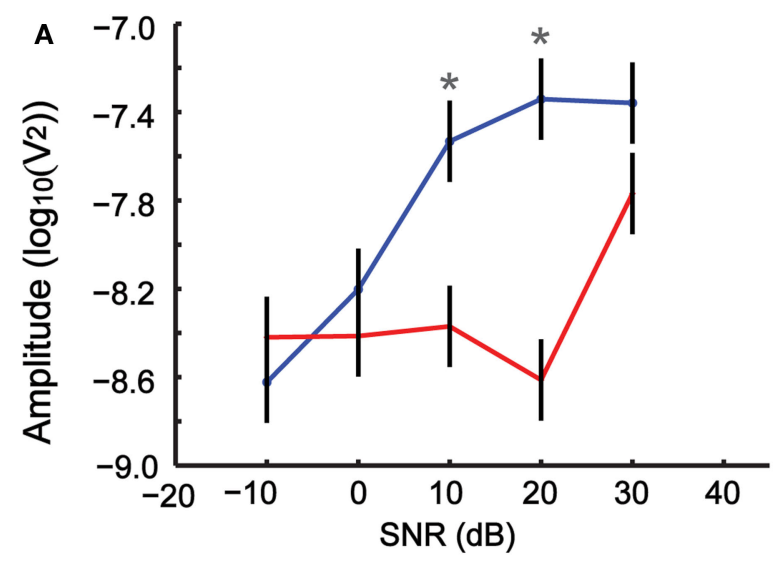

B
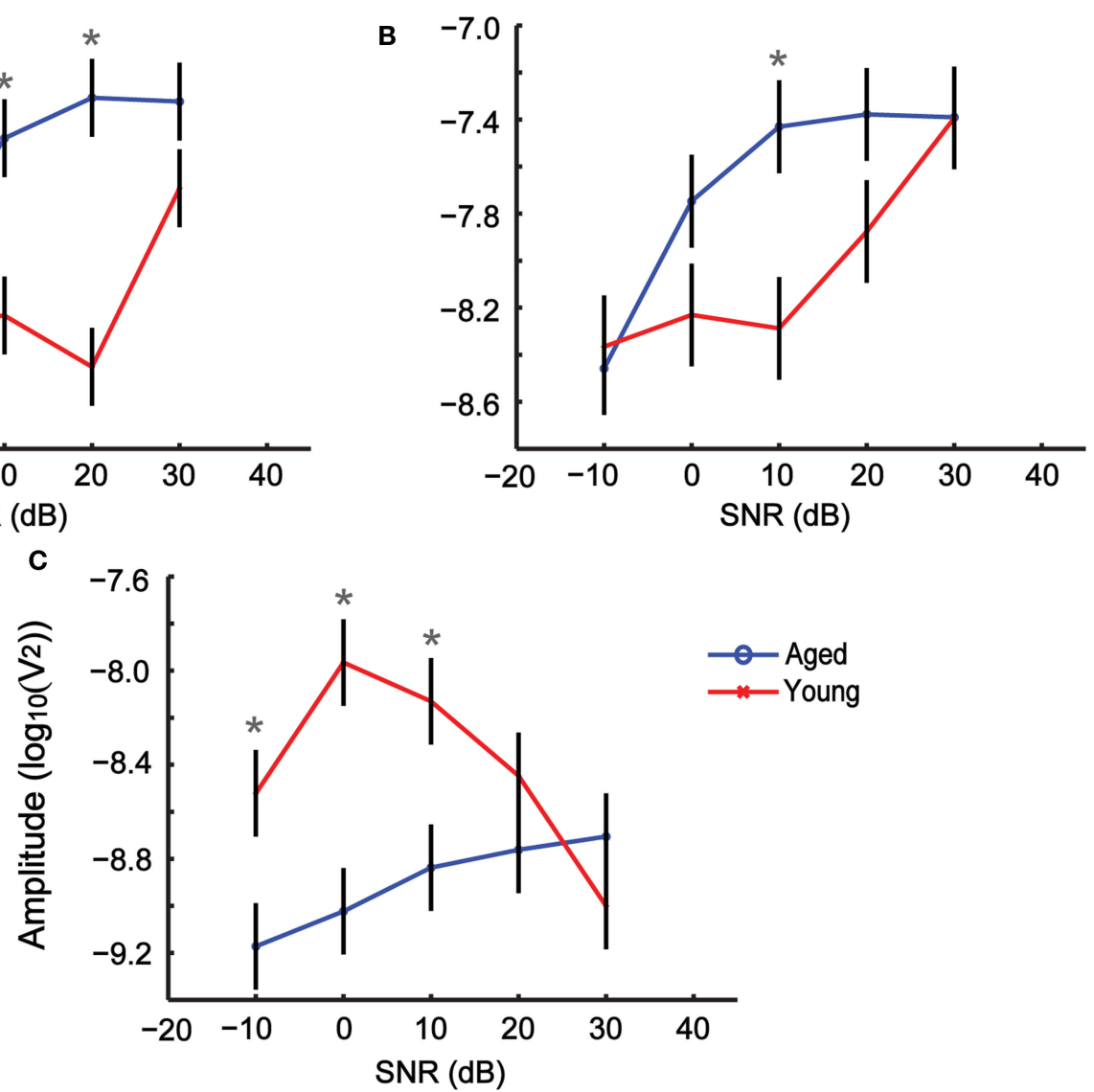

FIGURE 7 | Changes in AMFR amplitudes for SAM stimuli presented with simultaneous background noise are dependent on age, modulation frequency and signal-to-noise ratio (SNR). (A-C) Modulation frequencies
256, 512, and $1024 \mathrm{~Hz}$ respectively. The $x$ axis indicates SNR, and the $y$ axis indicates the LSM $\pm S E$ of the FFT amplitudes. Asterisks indicate statistically significant difference in LSMs $(P<0.05$, MANOVA). 
difficulty in speech recognition when the stimuli are time compressed to obtain faster modulation frequencies of the envelope (Versfeld and Dreschler, 2002; Grose et al., 2009). AMFRs from older adults are reduced at higher modulation frequencies when compared to younger adults (128 Hz in humans), but are similar at lower modulation frequencies (32 Hz) (Grose et al., 2009). This is also in accordance with responses from single neurons in the IC of rats to SAM stimuli in quiet, which show surprisingly small differences, with no change in modulation gain or the percentage of AM responsive units (Palombi et al., 2001) but a decrease in the percentage of synchronized units above $200 \mathrm{~Hz}$ in the aged, and an increase in overall spike count at lower modulation frequencies (Walton et al., 2002).

Stark differences emerge in our study when wideband background noise is added to the stimuli (Figure 7). At the lowest modulation frequency presented, $256 \mathrm{~Hz}$, the addition of moderate levels of background noise substantially reduces AMFR amplitudes in young but not in aged animals. A similar trend is observed, albeit to a lesser degree, at $512-\mathrm{Hz}$ modulation frequency where the aged have higher AMFR amplitudes than the young at SNR +10 , but are similar to the young at all other SNRs. When the modulation frequency is increased even further to $1024 \mathrm{~Hz}$, the trend reverses and the AMFR amplitudes of young animals are significantly higher than the aged at $\mathrm{SNR}+10$ and $\mathrm{SNR}+0$, where the AMFR amplitudes of the aged animals reduce significantly. This suggests that the addition of even a moderate amount of background noise degrades the phase locking ability in young animals, or drastically reduces the number of responsive neurons at 256 and $512 \mathrm{~Hz}$. At very rapid modulation frequencies such as $1024 \mathrm{~Hz}$, the addition of noise reduces responses from the aged animals to a greater degree than the young. These results are in agreement with human psychophysical studies where decrease in speech perception has been reported in older adults due to background noise (Dubno et al., 1984) and for higher noise interruption rates (Dubno et al., 2003).

In the young animals, the background noise is likely to drive neuronal excitation at the neurons' best frequency, as well as to engage inhibitory sidebands in the IC (Ehret and Moffat, 1985; LeBeau et al., 1996; Ramachandran et al., 2000; LeBeau et al., 2001) thereby reducing the response. In the case of reduced inhibition in the aged animals (reviewed in Caspary et al., 2008) or due to outer hair cell damage (Buckiova et al., 2007; Izquierdo et al., 2008; Chen et al., 2009; reviewed in Syka, 2010), the background noise may serve as non-specific excitation, thus allowing neurons with broader frequency response curves and higher thresholds to respond to the $256 \mathrm{~Hz}$ stimuli and maintain a strong AMFR. At the high modulation frequency of $1024 \mathrm{~Hz}$, it is unclear whether the larger responses in young animals are due to maintenance of temporal precision at high modulation frequencies or whether the modulation sidebands are recruiting more neurons in neighboring sharply tuned frequency bands in young animals (Hernandez et al., 2005). If the aged animals have a broader tuning curve due to reduction of inhibitory sidebands in the central auditory pathway (Palombi et al., 2001; Caspary et al., 2005; Schatteman et al., 2008) or due to outer hair cell damage (Chen et al., 2009; reviewed in Syka, 2010), the noise will produce non-specific excitation that will diminish the temporal precision of single neurons and therefore diminish the population
AMFR. It is also possible that there are sound level effects that differ between young and aged animals such that the increase in sound level by noise may boost the aged AMFR.

A few factors should be considered when interpreting results from this study. First, the presentation of the stimuli was done in free field, with the right ear of the animal facing the speaker. Similar conditions of recording were maintained for both young and aged animals, so the differences in AMFRs observed are not likely to be a result of the free field recordings. Second, all experiments have been performed under isoflurane anesthesia. Anesthesia alters auditory responses of single neurons to temporally complex stimuli at the level of the thalamus and the cortex, producing longer latency and less sustained responses (Ter-Mikaelian et al., 2007). Anesthesia is known to have a much lesser effect on responses from brainstem and midbrain nuclei like the IC (Szalda and Burkard, 2005; TerMikaelian et al., 2007), which are thought to be the major generators for the AMFRs (Kiren et al., 1994; Kuwada et al., 2002; Szalda and Burkard, 2005). Furthermore this study compares young and aged animals under similar conditions of anesthesia, with a lower amount of anesthesia for the aged to compensate for reduced liver function (Palombi and Caspary, 1996; Turner et al., 2005). Hence the observed differences in AMFR amplitudes cannot be attributed solely to the use of anesthesia. Third, the ABR thresholds for the young animals ranged between 35 and $55 \mathrm{~dB}$, the aged animals between 45 and $65 \mathrm{~dB}$ in this study. This is about 10-20 dB higher than the thresholds obtained in earlier studies performed using Long Evans or Fischer344 rats (Backoff and Caspary, 1994), but are similar to other studies using Fischer-344 rats (Popelar et al., 2006; reviewed in Syka, 2010). Those previous studies were primarily performed with stimuli presented using insert earphones in a single ear, using ketamine or pentobarbital anesthesia. The free field presentation of the stimuli, along with the isoflurane could account for the increased threshold that is seen in the ABR responses. And finally, all the AMFR stimuli presented used an $8-\mathrm{kHz}$ carrier frequency. This carrier frequency was empirically determined to provide the most robust AMFR, while having the least loss in tone ABRs, as well as minimal hair cell loss with age (reviewed in Syka, 2010).

There are various implications for this particular study, both in terms of further experiments, as well as from a diagnostic or clinical perspective. Although many studies of age-related changes in hearing measure ABR responses, few measure AMFR, especially in animal studies. Based on this study, we have demonstrated that AMFR in the aged F344 rat can reveal age-related changes in central auditory processing even when response threshold is compensated, particularly in the presence of background noise. These results also suggest that ABR thresholds and simple stimuli might not be sufficient in fully understanding the physiological correlates of changes in hearing with age in animals or in humans. Even when the aged listeners have normal behavioral or physiological audiograms, complex stimuli or stimuli presented under degraded listening conditions might better reveal processing deficits that would affect quality of life. Specifically, the use of sustained periodic stimuli may uncover age-related deficits in neural processing that cannot be obtained by studying phasic $A B R$ responses but that can be demonstrated in AMFR. This is consistent with known changes in single neuron representations of onset vs. sustained responses (Wang et al., 2005; Zheng and Escabi, 2008; Zhang and Kelly, 2010). Further studies are required to identify various 
sound stimuli that could tease out these processing deficits. Single unit studies in aged animals that show AMFR deficits could help us better understand the fine-grained neural representations behind them. Rigorous study of AMFRs could also help us bridge the gap between psychometric evaluations and single unit responses, as they provide an intermediate level of complexity where we can control and understand the acoustic features that are most affected with age.

\section{REFERENCES}

Backoff, P. M., and Caspary, D. M. (1994). Age-related-changes in auditory brain-stem responses in fischer- 344 rats-effects of rate and intensity. Hear. Res. 73, 163-172.

Backoff, P.M., Palombi, P. S., and Caspary, D. M. (1999). gamma-Aminobutyric acidergic and glycinergic inputs shape coding of amplitude modulation in the chinchilla cochlear nucleus. Hear. Res. 134, 77-88.

Boettcher, F. A., White, D. R., Mills, J. H., and Schmiedt, B. N. (1995). agerelated-changes in auditory-evoked potentials of gerbils.3. Low-frequency responses and repetition rate effects. Hear. Res. 87, 208-219.

Boettcher, F. A., Mills, J. H., Swerdloff, J. L., and Holley, B. L. (1996). Auditory evoked potentials in aged gerbils: responses elicited by noises separated by a silent gap. Hear. Res. 102, 167-178.

Boettcher, F. A., Poth, E. A., Mills, J. H., and Dubno, J. R. (2001). The amplitude-modulation following response in young and aged human subjects. Hear. Res. 153, 32-42.

Buckiova, D., Popelar, J., and Syka, J. (2007). Aging cochleas in the F344 rat: morphological and functional changes. Exp. Gerontol. 42, 629-638.

Caspary, D. M., Palombi, P.S., and Hughes, L. F. (2002). GABAergic inputs shape responses to amplitude modulated stimuli in the inferior colliculus. Hear. Res. 168, 163-173.

Caspary, D. M., Schatteman, T. A., and Hughes, L. F. (2005). Age-related changes in the inhibitory response properties of dorsal cochlear nucleus output neurons: role of inhibitory inputs. J. Neurosci. 25, 10952-10959.

Caspary, D. M., Arneric, S. P., Raza, A., and Finlayson, P. (1990a). Age-relatedchanges in the auditory-system of the fischer-344 rat. J. Am. Geriatr. Soc. 38, A15-A15.

Caspary, D. M., Raza, A., Armour, B. A. L., Pippin, J., and Arneric, S. P. (1990b). Immunocytochemical and neurochemical evidence for agerelated loss of gaba in the inferior colliculus - implications for neural presbycusis. J. Neurosci. 10, 2363-2372.

Caspary, D. M., Ling, L., Turner, J. G., and Hughes, L. F. (2008). Inhibitory neu- rotransmission, plasticity and aging in the mammalian central auditory system. J. Exp. Biol. 211, 1781-1791.

CHABA. (1988). Speech understanding and aging.99. J. Acoust. Soc. Am. 83, 859-895.

Chen, G. D., Li, M.N., Tanaka, C., Bielefeld, E. C., Hu, B. H., Kermany, M. H., Salvi, R., and Henderson, D. (2009). Aging outer hair cells (OHCs) in the Fischer 344 rat cochlea: function and morphology. Hear. Res. 248, 39-47.

Cunningham, J., Nicol, T., Zecker, S. G., Bradlow, A., and Kraus, N. (2001). Neurobiologic responses to speech in noise in children with learning improvement. Clin. Neurophysiol. 112, 758-767.

Dolphin, W. F., and Mountain, D. C. (1992). The envelope following response - scalp potentials elicited in the mongolian gerbil using sinusoidally am acoustic-signals. Hear. Res. $58,70-78$.

Dubno, J. R., Dirks, D. D., and Morgan, D. E. (1984). Effects of age and mild hearing-loss on speech recognition in noise. J. Acoust. Soc. Am. 76, 87-96.

Dubno, J. R., Horwitz, A. R., and Ahlstrom, J. B. (2003). Recovery from prior stimulation: masking of speech by interrupted noise for younger and older-adults with normal hearing. J. Acoust. Soc. Am. 113, 2084-2094.

Ehret, G., and Moffat, A. J. M. (1985). Inferior colliculus of the house mouse.2. Single unit responses to tones, noise and tone-noise combinations as a function of sound intensity. J. Comp. Physiol. Neuroethol. Sens. Neural Behav. Physiol. 156, 619-635.

Finneran, J. J., London, H. R., and Houser, D. S. (2007). Modulation rate transfer functions in bottlenose dolphins (Tursiops truncatus) with normal hearing and high-frequency hearing loss. J. Comp. Physiol. Neuroethol. Sens. Neural Behav. Physiol. 193, 835-843.

Foeller, E., Vater, N., and Kossl, M. (2001). Laminar analysis of inhibition in the gerbil primary auditory cortex. $J$. Assoc. Res. Otolaryngol. 2, 279-296.

Frisina, D. R., and Frisina, R. D. (1997). Speech recognition in noise and presbycusis: Relations to possible neural mechanisms. Hear. Res. 106, 95-104.

Frisina, R. D., and Walton, J. P. (2006) Age-related structural and functional problems: deficits and strategies for

\section{ACKNOWLEDGMENTS}

This study was supported by grants from the National Organization for Hearing Research and the American Federation for Aging Research (Edward L. Bartlett). Aravindakshan Parthasarathy was supported by the David Ross fellowship for 2008-09. The authors wish to thank Dr. Jeffrey Lucas for help with statistical analysis.

changes in the cochlear nucleus. Hear. Res. 216, 216-223.

Frisina, R. D., Smith, R. L., and Chamberlain, S. C. (1990a). Encoding of amplitude-modulation in the gerbil cochlear nucleus.1. A hierarchy of enhancement. Hear. Res. 44, 99-122.

Frisina, R. D., Smith, R. L., and Chamberlain, S. C. (1990b). Encoding of amplitude-modulation in the gerbil cochlear nucleus.2. Possible neural mechanisms. Hear. Res. 44, 123-141.

Grose, J. H., Mamo, S. K., and Hall, J. W. (2009). Age effects in temporal envelope processing: speech unmasking and auditory steady state responses. Ear Hear. 30, 568-575.

Hall, J. (2006). Handbook of Auditory Evoked Responses. Boston: Allyn \& Bacon.

He, N. J., Mills, J. H., Ahlstrom, J. B., and Dubno, J. R. (2008). Age-related differences in the temporal modulation transfer function with puretone carriers. J. Acoust. Soc. Am. 124, 3841-3849.

Helfert, R. H., Krenning, J., Wilson, .T S., and Hughes, L. F. (2003). Age-related synaptic changes in the anteroventral cochlear nucleus of Fischer-344 rats. Hear. Res. 183, 18-28.

Helfert, R. H., Sommer, T. J., Meeks, J., Hofstetter, P., and Hughes, L. F. (1999). Age-related synaptic changes in the central nucleus of the inferior colliculus of Fischer-344 rats. J. Comp. Neurol. 406, 285-298.

Henry, K. S., and Lucas, J. R. (2008). Coevolution of auditory sensitivity and temporal resolution with acoustic signal space in three songbirds. Anim. Behav. 76, 1659-1671.

Herdman, A. T., Lins, O., Van Roon, P., Stapells, D. R., Scherg, M., and Picton, T. W. (2002). Intracerebral sources of human auditory steadystate responses. Brain Topogr. 15 69-86.

Hernandez, O., Espinosa, N., PerezGonzalez, D., and Malmierca, M. S. (2005). The inferior colliculus of the rat: A quantitative analysis of monaural frequency response areas. Neuroscience 132, 203-217.

Hood, L. J. (1998). Clinical Applications of the Auditory Brainstem Response. San Diego, CA: Singular Publication Group.
Izquierdo, M. A., Gutierrez-Conde, P. M., Merchan, M. A., and Malmierca, M. S. (2008). Non-plastic reorganization of frequency coding in the inferior colliculus of the rat following noiseinduced hearing loss. Neuroscience $154,355-369$.

King, C., Warrier, C. M., Hayes, E., and Kraus, N. (2002). Deficits in auditory brainstem pathway encoding of speech sounds in children with learning problems. Neurosci. Lett. 319, 111-115.

Kiren, T., Aoyagi, M., Furuse, H., and Koike, Y. (1994). An experimentalstudy on the generator of amplitudemodulation following response. Acta Otolaryngol. 511, 28-33.

Krishnan, A. (2002). Human frequencyfollowing responses: representation of steady-state synthetic vowels. Hear. Res. 166, 192-201.

Kuwada, S., Anderson, J. S., Batra, R., Fitzpatrick, D. C., Teissier, N., and D'Angelo, W. R. (2002). Sources of the scalp-recorded amplitude-modulation following response. J. Am. Acad. Audiol. 13, 188-204.

LeBeau, F. E. N., Malmierca, M. S., and Rees, A. (2001). Iontophoresis in vivo demonstrates a key role for GABA(A) and glycinergic inhibition in shaping frequency response areas in the inferior colliculus of guinea pig. $J$. Neurosci. 21, 7303-7312.

LeBeau, F. E. N., Rees, A., and Malmierca, M.S. (1996). Contribution of GABAand glycine-mediated inhibition to the monaural temporal response properties of neurons in the inferior colliculus. J. Neurophysiol. 75, 902-919.

Leigh-Paffenroth, E. D., and Fowler, C. G. (2006). Amplitude-modulated auditory steady-state responses in younger and older listeners. J. Am. Acad. Audiol. 17, 582-597.

Ling, L. L., Hughes, L. F., and Caspary, D. M. (2005). Age-related loss of the GABA synthetic enzyme glutamic acid decarboxylase in rat primary auditory cortex. Neuroscience 132, 1103-1113.

Mazelova, J., Popelar, J., and Syka, J. (2002). "Auditory function in presbycusis: peripheral vs. central changes," in Sixth International Symposium on the Neurobiology and Neuroendocrinology of Aging (Bregenz, Austria: PergamonElsevier Science Ltd.), 87-94.

Meng, Q. Y., Wong, Y. T., Chen, J., and Ruan, R. S. (2007). Age-related 
changes in mitochondrial function and antioxidative enzyme activity in fischer 344 rats. Mech. Ageing Dev. 128, 286-292.

Mooney, T. A., Nachtigall, P. E., Taylor, K. A., Rasmussen, M. H., and Miller, L. A. (2009). Auditory temporal resolution of a wild white-beaked dolphin (Lagenorhynchus albirostris). J. Comp. Physiol. Neuroethol. Sens. Neural Behav. Physiol. 195, 375-384.

Palombi, P. S., and Caspary, D. M. (1996). Physiology of the young adult Fischer 344 rat inferior colliculus: Responses to contralateral monaural stimuli. Hear. Res. 100, 41-58.

Palombi, P. S., Backoff, P. M., and Caspary, D.M. (2001). Responses of young and aged rat inferior colliculus neurons to sinusoidally amplitude modulated stimuli. Hear. Res. 153, 174-180.

Picton, T. W., John, M. S., Dimitrijevic, A., and Purcell, D. (2003). Human auditory steady-state responses. Int. J. Audiol. 42, 177-219.

Popelar, J., Groh, D., Mazelova, J., and Syka, J. (2003). Cochlear function in young and adult Fischer 344 rats. Hear. Res. 186, 75-84.

Popelar, J., Groh, D., Pelanova, J., Canlon, B., and Syka, J. (2006). Age-related changes in cochlear and brainstem auditory functions in Fischer 344 rats. Neurobiol. Aging 27, 490-500.

Ramachandran, R., Davis, K. A., and May, B. J. (2000). Rate representation of tones in noise in the inferior colliculus of decerebrate cats. JARO 1, 144-160.

Rosen, S. (1992). Temporal information in speech - acoustic, auditory and linguistic aspects. Philos. Trans. R. Soc. Lond. B., Biol. Sci. 336, 367-373.

Russo, N., Nicol, T., Musacchia, G., and Kraus, N. (2004). Brainstem responses to speech syllables. Clin. Neurophysiol. 115, 2021-2030.

Russo, N. M., Nicol, T. G., Zecker, S. G., Hayes, E. A., and Kraus, N. (2005). Auditory training improves neural timing in the human brainstem. Behav. Brain Res. 156, 95-103.

Schatteman, T. A., Hughes, .L F., and Caspary, D. M. (2008). Aged-related loss of temporal processing: Altered responses to amplitude modulated tones in rat dorsal cochlear nucleus. Neuroscience 154, 329-337.

Schneider, B. A., Daneman, M., and Murphy, D. R. (2005). Speech comprehension difficulties in older adults: cognitive slowing or age-related changes in hearing? Psychol. Aging 20, 261-271.

Schneider, B. A., Pichorafuller, M. K., Kowalchuk, D., and Lamb, M. (1994). Gap detection and the precedence effect in young and old adults. $J$. Acoust. Soc. Am. 95, 980-991.

Snell, K. B., Ison, J. R., and Frisina, D. R. (1994). The effects of signal frequency and absolute bandwidth on gap detection in noise. J. Acoust. Soc. Am. 96, 1458-1464.

Starr, A., Picton, T. W., Sininger, Y., Hood, L. J., and Berlin, C. I. (1996). Auditory neuropathy. Brain 119, 741-753.

Strouse, A., Ashmead, D. H., Ohde, R. N., and Grantham, D. W. (1998). Temporal processing in the aging auditory system. J. Acoust. Soc. Am. 104, 2385-2399.

Syka, J. (2010). The Fischer 344 rat as a model of presbycusis. Hear. Res. 264 70-78.

Szalda, K., and Burkard, R. (2005). The effects of nembutal anesthesia on the auditory steady-state response (ASSR) from the inferior colliculus and auditory cortex of the chinchilla. Hear. Res. 203, 32-44.

Ter-Mikaelian, M., Sanes, D. H., and Semple, M.N. (2007). Transformation of temporal properties between auditory midbrain and cortex in the awake Mongolian gerbil. J. Neurosci. 27, 6091-6102.

Turner, J. G., Hughes, L. F., and Caspary, D. M. (2005). Divergent response properties of layer-V neurons in rat primary auditory cortex. Hear. Res. 202, 129-140.

Versfeld, N. J., and Dreschler,W.A. (2002) The relationship between the intelligibility of time-compressed speech and speech in noise in young and elderly listeners. J. Acoust. Soc. Am. 111, 401-408.

Walton, J. P., Simon, H., and Frisina, R. D. (2002). Age-related alterations in the neural coding of envelope periodicities. J. Neurophysiol. 88, 565-578.

Wang, J., Caspary, D., and Salvi, R. J. (2000). GABA-A antagonist causes dramatic expansion of tuning in primary auditory cortex. Neuroreport 11, 1137-1140.

Wang, J. A., McFadden, S. L., Caspary, D., and Salvi, R. (2002). Gammaaminobutyric acid circuits shape response properties of auditory cortex neurons. Brain Res. 944, 219-231.
Wang, X. Q., Lu, T., Snider, R. K., and Liang, L. (2005). Sustained firing in auditory cortex evoked by preferred stimuli. Nature 435, 341-346.

Zhang, H. M., and Kelly, J. B. (2010). Time dependence of binaural responses in the rat's central nucleus of the inferior colliculus. Hear. Res. 268, 271-280.

Zheng, Y., and Escabi, M. A. (2008). Distinct roles for onset and sustained activity in the neuronal code for temporal periodicity and acoustic envelope shape. J. Neurosci. 28, 14230-14244.

Conflict of Interest Statement: The authors declare that the research was conducted in the absence of any commercial or financial relationships that could be construed as a potential conflict of interest.

Received: 05 October 2010; paper pending published: 05 November 2010; accepted: 01 December 2010; published online: 17 December 2010

Citation: Parthasarathy A, Cunningham PA and Bartlett EL (2010) Age-related differences in auditory processing as assessed by amplitude-modulation following responses in quiet and in noise. Front. Ag. Neurosci. 2:152. doi: 10.3389/fnagi.2010.00152

Copyright (c) 2010 Parthasarathy, Cunningham and Bartlett. This is an open-access article subject to an exclusive license agreement between the authors and the Frontiers Research Foundation, which permits unrestricted use, distribution, and reproduction in any medium, provided the original authors and source are credited. 